\title{
Beneficios de la implantación de la Marca Q de turismo industrial
}

\author{
Silvia Portela Maquieira* \\ Manuel Martínez Carballo** Eduardo Guillén Solórzano*** \\ Universidade da Coruña (España)
}

\begin{abstract}
Resumen: A diferencia de lo que pasaba hace años en los que no se valoraba la calidad en las empresas debido a que la demanda era mayor que la oferta, hoy en día ocurre todo lo contrario y la calidad se ha convertido en una de las variables claves de competitividad que ha pasado de aplicarse en los procesos productivos a ámbitos tan complejos como puede ser en el sector turístico.

En este último campo se centra el contenido de este trabajo. En primer lugar, y a modo de introducción, se analizan ciertos aspectos del Turismo Industrial; posteriormente, se estudia la importancia de la Marca Q de Calidad Turística, en tercer lugar, se describe la investigación empírica llevada a cabo y, finalmente, se determinan los factores claves sobre la implantación de esta Marca de Calidad en las empresas españolas certificadas.
\end{abstract}

Palabras Clave: Calidad Turística, Certificación, UNE 302001:2012, ICTE, Diferenciación, Turismo Industrial.

\section{Benefits of implementation of industrial tourism Q Brand}

Abstract: Some years ago the quality of the companies was not valued since demand was higher than the offer but, today, this situation has changed due to the quality has become in one of the most important variable competitiveness key, which, has applied in productive process as well as complex ambits such as tourism sector.

This field is the main content of this paper that are structured into four distinct parts centers.

Firstly and, as an introduction way, certain aspects of Industrial Tourism are analyzed. Subsequently, the importance of the Q Mark for Quality is studied. Thirdly, empirical research that has been studied is described and, finally, the key factors on the implementation of the Quality Mark.

Keywords: Tourism Quality Certification UNE 302001:2012, ICTE, Differentiation, Industrial Tourism.

\section{Contextualización}

El turismo es el sector emblemático de la economía española. Sin embargo, su evolución global no resulta equilibrada en la medida que ciertos subsectores experimentan un desarrollo notable y continuado en el tiempo mientras otros mantienen un carácter residual. Éste es el caso del turismo industrial, una oferta de turismo cultural que muestra un potencial de desarrollo muy superior al actual.

Existen diversidad de opiniones sobre la noción y alcance del turismo industrial. En la Norma UNE 302001:2012 se interpreta en un sentido amplio, tratando de dar cabida a las diferentes manifestaciones de una compleja realidad.

Esta es una rama emergente de la actividad turística que se traduce en visitas y actividades diversas en lugares industriales, representativos del pasado y del presente de la cultura asociada al sistema productivo.

\footnotetext{
* E-mail: silvia.pmaquieira@udc.es

** E-mail: mmc@udc.es

*** E-mail: edugs@udc.es
} 
Para el destino, el turismo industrial es una manera de diversificar la oferta turística al mismo tiempo que pone en valor al territorio, a sus organizaciones empresariales y a su sociedad. De esta manera, se consigue aumentar el atractivo del destino turístico en su conjunto dándole un valor diferencial.

Con el turismo industrial se obtienen beneficios, principalmente promocionales y de imagen social, que sirven como elementos integradores del territorio. Por tanto, el turismo industrial es sinónimo de innovación, factor que constituye un aspecto clave para el éxito de los lugares y organizaciones implicados en este sector.

La Norma UNE 302001:2012 pretende ser un estímulo para fomentar la puesta en marcha de actividades de turismo industrial al ofrecer a las organizaciones de manera indirecta una guía de actuación. Si la innovación y la calidad se han convertido en las claves del éxito de las organizaciones en el siglo XXI, las actividades de turismo industrial se configuran como un potente elemento innovador que abre una nueva línea de actividad para las organizaciones, ya que, aumenta su visibilidad y plantea una estrategia de diversificación que puede incrementar sus beneficios (Norma UNE 302001:2012).

El usuario tendrá la oportunidad de disfrutar de una doble experiencia. Por un lado, experimentará una actividad lúdica y, por otro, podrá conocer en primera persona el saber hacer y los productos de las organizaciones.

La Norma ha querido ser accesible a cualquier tipo de empresa, independientemente de su actividad o el tamaño de su organización, porque tradicionalmente estas actividades han venido circunscribiéndose a organizaciones de una cierta dimensión.

El alcance de la Norma ha buscado abarcar un escenario más amplio al englobar no solo la actividad de carácter básico y visita-guiada o autoguiada por el lugar industrial, (que tiene como finalidad última transmitir fielmente la cultura industrial del territorio y de sus organizaciones) sino también la oferta de servicios complementarios que aportan valor a la experiencia global del visitante.

En definitiva, esta es una Norma dirigida a aquellos turistas curiosos que buscan una experiencia diferente y a aquellas empresas abiertas a mostrar su historia, sus instalaciones y su producto (www. turismoindustrial.org).

\section{Marco teórico}

\subsection{La calidad en la política turística española}

El Sistema de Calidad Turístico Español fue creado por iniciativa del sector empresarial y con el apoyo de la Administración Central, en concreto, la Secretaria General de Turismo. Este sistema se ha convertido en la mayor apuesta de los últimos años en la mejora de la gestión interna y ésta repercute en la calidad del servicio. Es la estructura técnica e institucional que agrupa a los principales subsectores turísticos españoles (www.icte.es).

La Marca de Calidad Turística Española representada en la actualidad por la imagen de una Q, es una marca de garantía que se caracteriza por ser rigurosa, ya que, para obtenerla se hace imprescindible superar pruebas de carácter objetivo establecidas en las normas; prestigiosa, porque demuestra el compromiso empresarial de alcanzar la plena satisfacción del cliente; diferenciadora, en el sentido de que únicamente pueden obtenerla aquellos establecimientos que aseguren el cumplimiento de unos niveles mínimos de servicio y; por último, fiable porque los mecanismos utilizados para evaluar el cumplimiento o no de los requisitos de las normas son independientes del sector y se diseñan de acuerdo con la normativa intersectorial aplicable (Foronda y García, 2009)

Esta marca de garantía surgió por la necesidad de aplicar un sistema de gestión homogéneo a todo el sector turístico español y que hoy en día es coordinada por el Instituto para la Calidad Turística Española (ICTE) como único gestor que nació con el apoyo de la Administración Turística del Estado (Gonzalo, 2009). Este organismo español, privado, independiente y sin ánimo de lucro tiene como funciones básicas: la normalización, implantación, certificación y promoción de la Marca Q de Calidad Turística Española.

La implantación y/o posterior certificación de esta Marca Q de Calidad Turística sirve además como una herramienta de autoevaluación, ya que, las normas en las que se basa requieren un análisis exhaustivo de todas las áreas del establecimiento en las que se realiza un doble análisis basado en el sistema de calidad y en las correspondientes comprobaciones físicas. Por otra parte, si se aplica de manera recurrente en el tiempo, proporciona una información muy valiosa sobre la efectividad de las medidas correctoras implantadas para mejorar los puntos débiles, así como de la eficacia conseguida a la hora de reforzar las áreas más positivas (Carrasco, 2009). 
En el entorno actual la certificación de un sistema de gestión de la calidad ya no representa una ventaja competitiva, sino que esta fase debe entenderse como un proceso de mejora continua que nos llevará al camino de la Excelencia. Lo que sí está claro es, que una empresa turística española que implante un sistema de gestión de la calidad mediante esta marca de garantía obtendrá una serie de ventajas; por un lado, están las ventajas para la empresa como, por ejemplo, la mejora del producto o servicio, instrumento para la promoción y comercialización y, por otro lado, están las ventajas para el cliente como puede ser la disminución de la incertidumbre ante la elección del establecimiento turístico, confianza, garantía, etc... (Yepes, 2009).

No obstante, al ser una marca de garantía (algo particular de nuestro derecho interno) existe un inconveniente principal: la falta de reconocimiento de este distintivo de calidad, tanto del consumidor internacional como por parte de los operadores extranjeros, a diferencia de lo que ocurriría con la certificación ISO 9000 que es reconocida internacionalmente.

Por ello, aquellas empresas turísticas que reciban un alto porcentaje de clientes extranjeros deberían certificar su sistema de gestión de la calidad basado en una norma internacional como podría ser la familia de Normas ISO 9000 o incluso situarse en el última etapa de la evolución histórica del término calidad, que es la calidad total, mediante la obtención de la Excelencia a través del Modelo EFQM (Membrado, 2002).

En este sentido, el ICTE señala que las Normas Turísticas de Calidad se sitúan en un nivel intermedio entre las Normas ISO 9000 y el Modelo EFQM de Excelencia, por lo que la implantación de la Marca Q de Calidad Turística es perfectamente compatible con una certificación ISO 9000 y la Excelencia, estando la diferencia entre ambas certificaciones en los requisitos aplicables y el nivel de exigencia de las Normas de referencia. Algunas Comunidades Autónomas están realizando un estudio para ver la compatibilidad de poder certificar conjuntamente en una empresa turística la Marca Q y la ISO 9000.

Por último, indicar que el proceso de adhesión y certificación de la Marca $\mathrm{Q}$, se rige por un proceso secuencial de actividades relativas tanto a la entidad turística como al ente gestor (ICTE) y, que de forma muy breve, se puede resumir en el siguiente gráfico.

\section{Gráfico 1: Proceso de adhesión y certificación de la Marca Q de Calidad Turística}

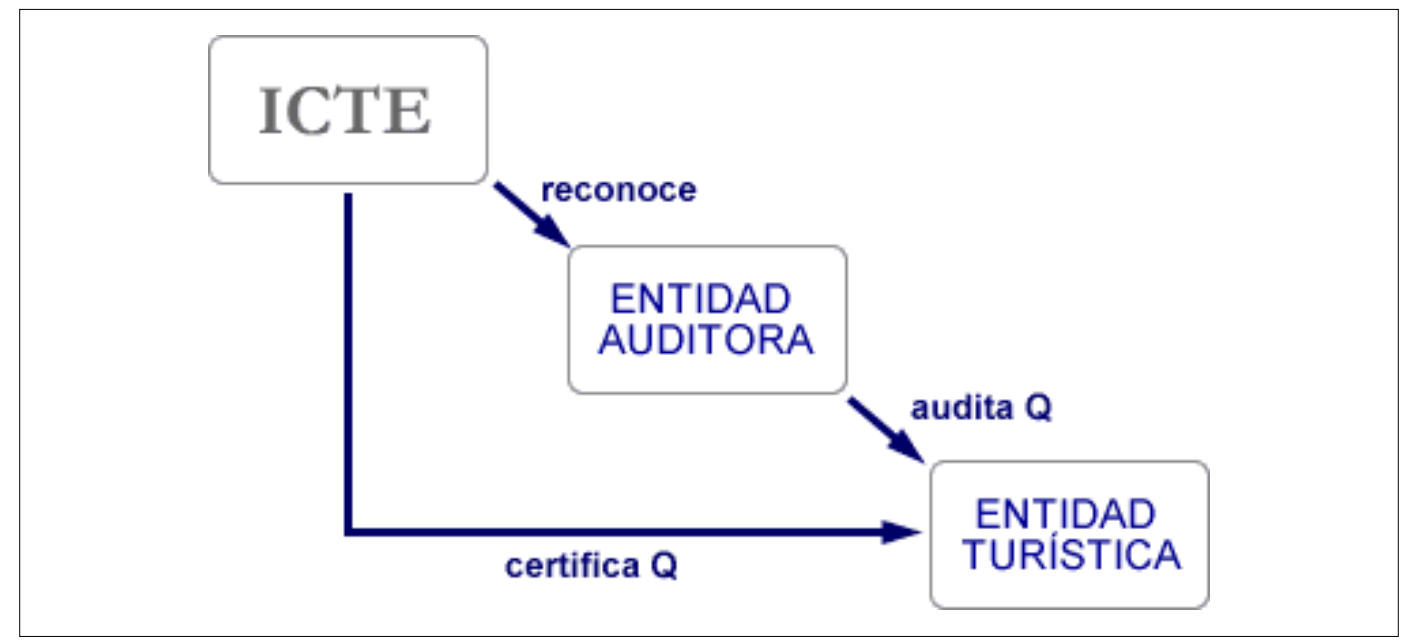

Fuente: Instituto para la Calidad Turística Española

\subsection{La marca q de calidad turística en las empresas españolas}

En el año 2012 se publico la Norma UNE 302001, que permite a las instalaciones industriales certificar bajo la prestigiosa Marca Q del Instituto para la Calidad Turística Española (ICTE). Esto brinda a las industrias una oportunidad para potenciar su diversificación mediante el turismo enológico. Marcando su diferenciación, liderazgo y promoción. Siendo pioneros en este campo y explotando más y mejor el 
reclamo del turismo industrial, como fuente de ingresos directa y como canal eficaz para la difusión y comercialización de su marca y de puesta en valor la excelencia de su industria o bodega.

Esta certificación está teniendo una excelente aceptación por parte del sector. Ya son numerosas las bodegas y empresas con la Calidad de su oferta Turística Industrial certificada y muchas otras las que están en proceso. El momento contextual en el que se actúe es determinante en los resultados de cualquier iniciativa. Precisamente es ahora el tiempo y el contexto idóneo para acometer esta implantación y certificación y pertenecer al grupo de "los primeros". Y de este modo, sacar el mayor rédito comercial y publicitario, tanto por parte de su organización como por la promoción y difusión que el ICTE está llevando a cabo.

Durante el año 2012, el Instituto de Calidad Turística otorgó por primera vez la Q de Calidad en la categoría de turismo industrial. En total, se han otorgado veintitrés certificaciones en toda España hasta la fecha de las cuales. La consecución de la Q de Calidad supone no sólo la certificación de la idoneidad de las instalaciones, sino también la calidad de los servicios turísticos prestados por estos establecimientos enoturísticos, ya que, el ICTE ha valorado las características de las instalaciones propias de estos espacios incluyendo su gestión interna, los servicios prestados y sus objetivos de calidad con el fin de mostrar sus procesos y su saber hacer, generando experiencias basadas en la actividad industrial, científica y técnica del presente y del pasado.

\section{Investigación realizada}

\subsection{Objetivos}

El objetivo de la investigación es conocer los beneficios que ha reportado la Marca Q de Turismo Industrial a estas empresas. La finalidad última del trabajo es conocer los beneficios conseguidos y valorar cuáles de ellos han resultado más significativos para las empresas. La implantación y/o certificación de la $\mathrm{Q}$ de Calidad Turística es una potente y práctica herramienta que, en teoría, reporta numerosas ventajas en la gestión de éste recurso, entre los cuales destacan:

1) Diferenciación respecto a los competidores.

2) Promoción y difusión, en especial para los pioneros.

3) La mejora en los procesos de gestión.

4) Satisfacción del visitante.

5) Efecto bumerán y exponencial del número de visitas.

6) Detección de problemas y mejora continua.

7) Cuota de mercado.

8) Beneficios empresariales.

Para conocer los verdaderos beneficios que las empresas han obtenido con la certificación se han analizados estos 8 ítems.

\subsection{Metodología empleada}

En este apartado se describe la metodología para llevar a cabo el estudio empírico de la investigación definida anteriormente. Este trabajo se ha centrado en la Marca Q de Calidad Turística por ser el certificado de calidad más novedoso obtenido por los establecimientos turísticos de nuestro país.

La metodología empleada se puede resumir en las siguientes etapas. En primer lugar, se ha procedido a realizar una base de datos "ad-hoc" con todas las empresas con esta Marca de Calidad a partir de la información obtenida de la página Web del ICTE (www.icte.es). Como segunda etapa, se elaboró una encuesta sobre los beneficios obtenidos con la consecución de los requisitos y posterior certificación, entre otros aspectos. Finalmente, se envió la encuesta a la totalidad de las entidades almacenadas en la base de datos mediante correo electrónico o correo ordinario y, posteriormente, se realizó la tabulación de las mismas y se extrajeron los resultados.

Las preguntas iniciales establecen el perfil de las empresas que se han encuestado en base a su año de constitución, número de trabajadores en plantilla y facturación anual. También se han realizado preguntas sobre el tiempo dedicado a la implantación y certificación, así como al apoyo externo de consultorías y empresas certificadoras. Una vez establecido el perfil se les pregunta sobre los beneficios en diferentes factores (imagen, gestión, resultados económicos, etc.) que la certificación les ha podido reportar. 
En la siguiente tabla se recogen las preguntas planteadas en el cuestionario. Se trata de 8 cuestiones que cada una de las entidades tuvo que valorar en una escala de 1 a 5 ; donde el 1 significa "Ha empeorado", el 2 significa "No ha mejorado o se mantiene / Indiferente", el 3 "Ha mejorado un poco", el 4 "Ha mejorado bastante", y el 5 "Ha mejorado mucho".

Tabla 1: Preguntas del cuestionario y escala de valorac

\begin{tabular}{|c|c|}
\hline Pregunta: & Escala: \\
\hline P1. La imagen de la empresa & $\square_{1} \square_{2} \square_{3} \square_{4} \square_{5}$ \\
\hline P2. La diferenciación de nuestra empresa respecto a los demás competidores & $\square_{1} \square_{2} \square_{3} \square_{4} \square$ \\
\hline P3. La prestación o realización del servicio & $\square_{1} \square_{2} \square_{3} \square_{4} \square_{5}$ \\
\hline P4. El servicio de atención al cliente & ${ }_{2} \square_{3} \square_{4} \square_{5}$ \\
\hline P5. La eficacia de las tareas cotidianas o habituales de la empresa & $\square_{1} \square_{2} \square_{3} \square_{4} \square_{5}$ \\
\hline P6. La detección de problemas internos y áreas de mejora & $\square_{1} \square_{2} \square_{3} \square_{4} \square_{5}$ \\
\hline P7. La cuota de mercado & $\square_{1} \square_{2} \square_{3} \square_{4} \square_{5}$ \\
\hline P8. Los beneficios empresariales & $\square_{1} \square_{2} \square_{3} \square_{4} \square_{5}$ \\
\hline \multicolumn{2}{|c|}{$\begin{array}{c}\text { Valore de } 1 \text { a } 5 \text { el impacto que ha tenido la implantación y certificación de la Marca Q de Calidad } \\
\text { Turística de Turismo Industrial en los siguientes factores o aspectos: }\end{array}$} \\
\hline
\end{tabular}

Las preguntas se pueden organizar en cuatro bloques bien diferenciados:

- Primer bloque: P1 y P2 se centran en los motivos que versan sobre la imagen de la empresa y diferenciación.

- Segundo bloque: P3 y P4 tratan la prestación del servicio y atención al cliente.

- Tercer bloque: P5 y P6 se basan en cuestiones de mejora en la gestión.

- Cuarto bloque: P7 y P8 estudian la cuota de mercado y los beneficios empresariales.

El trabajo de campo de esta investigación empírica se ha realizado durante los meses de julio a septiembre de 2014 .

\subsection{Perfil de las empresas y de la muestra}

Según dos datos obtenidos la web del ICTE, a fecha 01/07/2014, las veintitrés empresas que han obtenido este reconocimiento son:

- Bodegas Terras Gauda (Pontevedra)

- Bodegas Palacio de Fefiñanes (Pontevedra)

- Bodegas Marqués de Vizhoja (Pontevedra)

- Bodegas Coto Redondo-Señorío de Rubiós (Pontevedra)

- Adega Eidos (Pontevedra)

- Bodegas Martín Códax (Pontevedra)

- Bodegas Don Olegario (Pontevedra)

- Bodegas Zárate (Pontevedra)

- Pazo de Señorans (Pontevedra)

- Adega Cooperativa Vitivinícola do Ribeiro (Ourense)

- Cooperativa San Roque (Ourense)

- Abadia Retuerta (Valldolid)

- Bodegas Beronia (La Rioja) 
- Bodegas Campo Viejo (La Rioja)

- Bodegas Protos (Valladolid)

- Bodegas Tio Pepe (Cadiz)

- Bodegas Ysios (Alava)

- Cune (La Rioja)

- Bodegas Martinez Lacuesta (La Rioja)

- Bodegas Eminia (Valladolid)

- Bodegas Pedro Benito Urbina (La Rioja)

- Estancia de Piedra (Zamora)

- Vivanco (La Rioja)

La encuesta ha logrado una buena acogida, ya que, todas las empresas certificadas con la Marca $\mathrm{Q}$ de Turismo Industrial han participado en ella.

El perfil de la muestra es muy diverso puesto que entre las bodegas que participan en este estudio existe una gran diversidad en cuanto a su año de fundación, siendo éste un valor poco homogenizado, en la que algunas bodegas cuentan con más de 100 años de antigüedad y otras son relativamente jóvenes. La de más reciente creación ha iniciado su actividad hace 10 años.

También hay diversidad en los datos sobre número de empleados y facturación anual.

Tabla 2: Datos de plantilla y facturación (2013)

\begin{tabular}{|c|c|}
\hline Facturación en millones de euros (2013) & Porcentaje \\
\hline DE 0 A 2 & $39,1 \%$ \\
\hline DE 2 A 10 & $26,1 \%$ \\
\hline DE 10 A 50 & $13 \%$ \\
\hline MAS DE 50 & $21,7 \%$ \\
\hline
\end{tabular}

\begin{tabular}{|c|c|}
\hline Empleados & Porcentaje \\
\hline DE 0 A 9 & $39,1 \%$ \\
\hline DE 10 A 49 & $8,7 \%$ \\
\hline DE 50 A 249 & $47,8 \%$ \\
\hline MAS DE 250 & $4,3 \%$ \\
\hline
\end{tabular}

Observando los datos obtenidos se puede decir que el 47,8\% de las bodegas encuestadas tienen menos de 50 empleados y el 34,7\% ha facturado más de 50 millones de euros en el año 2013.

Independientemente de las características de cada bodega, la calidad es un tema de importancia en todas ellas, así como la innovación y la adecuación de sus procedimientos a las demandas del sector.

En la siguiente tabla se observa la duración del proceso de certificación para poder cuantificar el tiempo dedicado a este proceso y la fecha en la que han obtenido la Marca Q de calidad turística.

Tabla 3: Tiempo dedicado a la certificación y fecha de obtención

\begin{tabular}{|c|c|}
\hline Tiempo dedicado a la certificación & Porcentaje \\
\hline MENOS DE 6 MESES & $60,9 \%$ \\
\hline ENTRE 6 Y 12 MESES & $30,4 \%$ \\
\hline MAS DE 12 MESES & $8,7 \%$ \\
\hline
\end{tabular}

A la vista de esta información, se observa que el proceso ha sido relativamente corto, casi el $61 \%$ de las bodegas han dedicado a esta gestión menos de 6 meses.

En cuanto a la ayuda o colaboración externa, ya sea a través de consultoría externa privada, consultoría externa gratuita, apoyo de otra empresa con experiencia, etc, para estudiar la forma en la que las bodegas han llevado a cabo la gestión de la certificación, la respuesta obtenida revela que el $91 \%$ de las empresas han utilizado apoyo externo para implantar los requisitos de la Marca Q de Turismo Industrial.

Por ejemplo, en el caso de las empresas de Galicia, este apoyo externo ha sido facilitado a todas ellas por la misma entidad consultora. 
Tabla 4: Datos sobre valoración del apoyo recibido en la gestión de la $\mathbf{Q}$

\begin{tabular}{|c|c|}
\hline Valoración & Porcentaje \\
\hline REGULAR & $4,3 \%$ \\
\hline BUENO & $60,9 \%$ \\
\hline MUY BUENO & $34,8 \%$ \\
\hline
\end{tabular}

Más de un $95 \%$ de las empresas valoró como bueno o muy bueno el servicio de apoyo externo mientras un $4,3 \%$ lo valoró como regular.

También se ha preguntado sobre la valoración de la entidad certificadora que le ha verificado el cumplimiento de los requisitos de la Marca Q de Turismo Industrial. En esta ocasión, casi un 48\% lo ha valorado como bueno y un $39 \%$ como muy bueno.

Tabla 5: Datos sobre la valoración de la entidad certificadora

\begin{tabular}{|c|c|}
\hline Valoración & Porcentaje \\
\hline REGULAR & $13 \%$ \\
\hline BUENO & $47,8 \%$ \\
\hline MUY BUENO & $39,1 \%$ \\
\hline
\end{tabular}

\section{Resultados obtenidos}

En la parte central del estudio se analizan los beneficios de la implantación/certificación de la Marca $\mathrm{Q}$ de Calidad Turística. A la luz de los resultados obtenidos, se observan diversos motivos que se han organizado en cuatro bloques diferenciados:

Primer Bloque: Motivos basados en la imagen de la empresa y la diferenciación (P1 y P2).

Segundo Bloque: Motivos basados en el servicio y atención al cliente (P3 y P4).

Tercer Bloque: Motivos basados en la mejora de la gestión (P5 y P6).

Cuarto Bloque: Motivos basados en la cuota de mercado y beneficios empresariales (P7 y P8).

\subsection{Primer bloque: los motivos basados en la imagen de la empresa y su diferenciación}

Con los resultados obtenidos se puede declarar que la imagen de las empresas certificadas ha mejorado bastante tras la implantación de la Marca Q; así lo afirma un 44\% de las empresas encuestadas.

La mayoría de estas empresas considera que la diferenciación de las empresas con respecto a los demás competidores ha mejorado con la consecución de la Marca, ya que, un 31\% cree que ha mejorado un poco, un $30 \%$ cree que ha mejorado bastante y un $13 \%$ cree que ha mejorado mucho. No obstante, un $4 \%$ de las empresas cree que ha empeorado en esta faceta.

Tabla 6: Motivos basados en la imagen y su diferenciación

\begin{tabular}{|c|c|c|c|c|c|}
\hline Valoraciones de la empresa & $\begin{array}{c}\text { Ha } \\
\text { empeorado }\end{array}$ & Indiferente & $\begin{array}{c}\text { Ha } \\
\text { mejorado } \\
\text { un poco }\end{array}$ & $\begin{array}{c}\text { Ha } \\
\text { mejorado } \\
\text { bastante }\end{array}$ & $\begin{array}{c}\text { Ha } \\
\text { mejorado } \\
\text { mucho }\end{array}$ \\
\hline P1. La imagen de la empresa & $0 \%$ & $26 \%$ & $26 \%$ & $44 \%$ & $4 \%$ \\
\hline $\begin{array}{c}\text { P2. La diferenciación de nuestra } \\
\text { empresa respecto a los demás } \\
\text { competidores }\end{array}$ & $4 \%$ & $22 \%$ & $31 \%$ & $30 \%$ & $13 \%$ \\
\hline
\end{tabular}


Gráfico 2: Imagen de la empresa

\begin{tabular}{|ll|}
\hline \multicolumn{2}{|c|}{ La imagen de la empresa } \\
口Ha empeorado \\
\\
\end{tabular}

Gráfico 3: Diferenciación con los competidores

La diferenciación de nuestra empresa respecto a los demás competidores

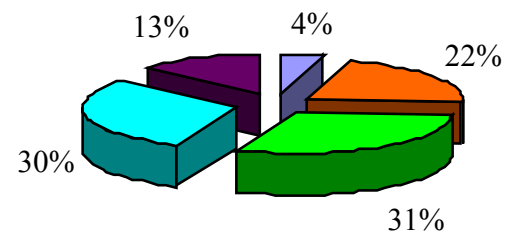

$\square$ Ha empeorado

$\square$ Indiferente

$\square$ Ha mejorado un poco

$\square$ Ha mejorado bastante

$\square$ Ha mejorado mucho

\subsection{Segundo bloque: los motivos sobre el servicio prestado y la atención al cliente}

En cuanto a la prestación del servicio hay un amplio consenso sobre la mejoría que ha supuesto la implantación de la Marca, ya que, un $35 \%$ cree que ha mejorado un poco, un $52 \%$ cree que ha mejorado bastante y un $9 \%$ cree que ha mejorado mucho.

Las empresas han opinado sobre la mejoría del servicio de atención al cliente: un $17 \%$ considera que ha mejorado un poco, un $65 \%$ que ha mejorado bastante y un $9 \%$ dice que ha mejorado mucho. En resumen, el 91\% de las empresas valoran que el servicio de atención al cliente ha mejorado con la implantación de la Marca Q.

Tabla 7. Motivos sobre el servicio y la atención al cliente

\begin{tabular}{|c|c|c|c|c|c|}
\hline Valoraciones de la empresa & $\begin{array}{c}\text { Ha } \\
\text { empeorado }\end{array}$ & Indiferente & $\begin{array}{c}\text { Ha } \\
\text { mejorado } \\
\text { un poco }\end{array}$ & $\begin{array}{c}\text { Ha } \\
\text { mejorado } \\
\text { bastante }\end{array}$ & $\begin{array}{c}\text { Ha } \\
\text { mejorado } \\
\text { mucho }\end{array}$ \\
\hline $\begin{array}{c}\text { P3. La prestación o realización } \\
\text { del servicio }\end{array}$ & $0 \%$ & $4 \%$ & $35 \%$ & $52 \%$ & $9 \%$ \\
\hline $\begin{array}{c}\text { P4. El servicio de atención al } \\
\text { cliente }\end{array}$ & $0 \%$ & $9 \%$ & $17 \%$ & $65 \%$ & $9 \%$ \\
\hline
\end{tabular}




\section{Gráfico 4: La prestación del servicio}

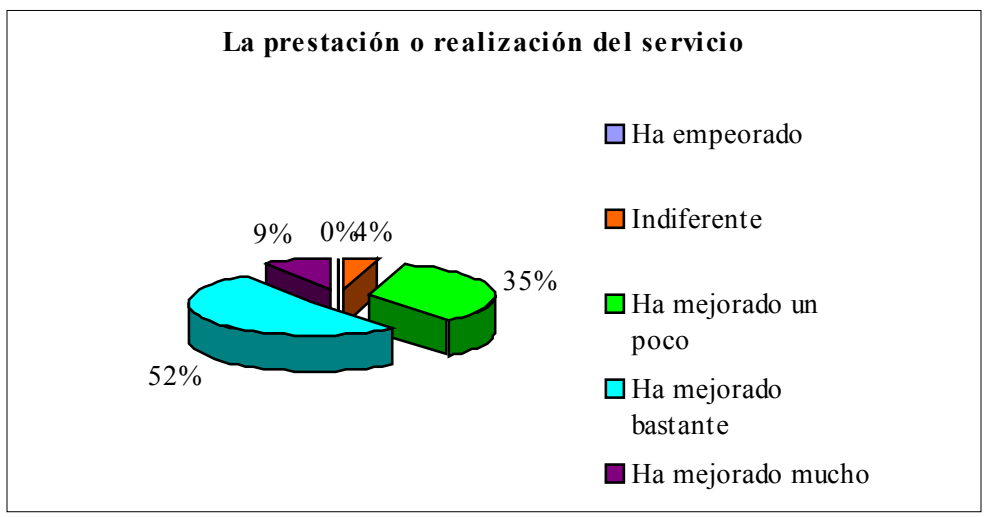

\section{Gráfico 5: La atención al cliente}

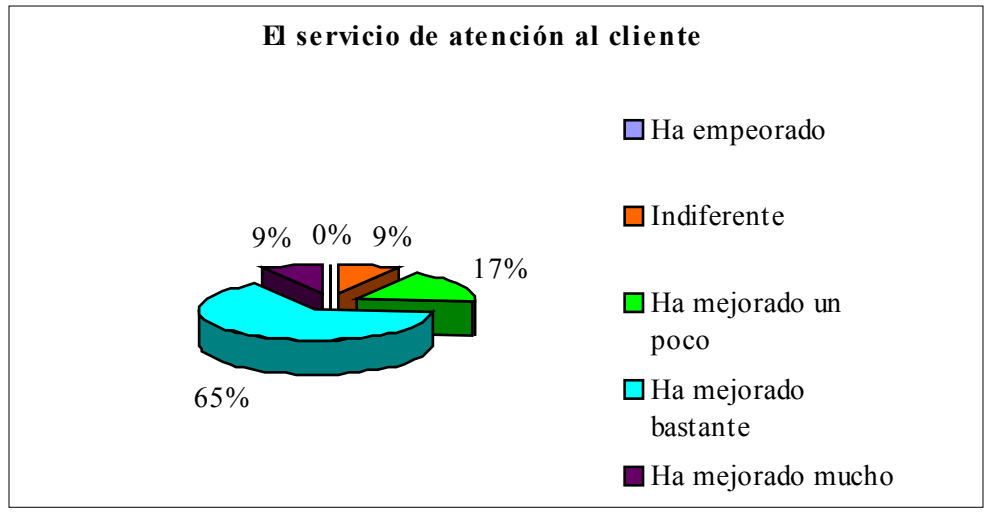

\subsection{Tercer bloque: la mejora en la gestión}

En cuanto a la mejora de la eficacia en las tareas cotidianas o habituales de la empresa las respuestas obtenidas ofrecen un amplio abanico, entre el $4 \%$ que cree que ha empeorado la eficacia, el $26 \%$ que no han notado mejoría, un $21 \%$ que han mejorado un poco, un $33 \%$ de las empresas cree que ha mejorado bastante y un $9 \%$ opinan que ha mejorado mucho.

En el área de detección de problemas internos la implantación de la Marca ha sido una mejora para casi todas las empresas encuestadas, ya que, sólo al 13\% le ha resultado indiferente y al $87 \%$ restante les ha sido de ayuda en mayor o menor medida.

Tabla 8: Motivos basados en la mejora de la gestión

\begin{tabular}{|c|c|c|c|c|c|}
\hline Valoraciones de la empresa & $\begin{array}{c}\text { Ha } \\
\text { empeorado }\end{array}$ & Indiferente & $\begin{array}{c}\text { Ha } \\
\text { mejorado } \\
\text { un poco }\end{array}$ & $\begin{array}{c}\text { Ha } \\
\text { mejorado } \\
\text { bastante }\end{array}$ & $\begin{array}{c}\text { Ha } \\
\text { mejorado } \\
\text { mucho }\end{array}$ \\
\hline $\begin{array}{c}\text { P5. La eficacia de las tareas } \\
\text { cotidianas o habituales de la } \\
\text { empresa }\end{array}$ & $4 \%$ & $26 \%$ & $21 \%$ & $39 \%$ & $9 \%$ \\
\hline $\begin{array}{c}\text { P6. La detección de problemas } \\
\text { internos y áreas de mejora }\end{array}$ & $0 \%$ & $13 \%$ & $35 \%$ & $43 \%$ & $9 \%$ \\
\hline
\end{tabular}




\section{Gráfico 6: La eficacia de las tareas}

\begin{tabular}{|l}
$\begin{array}{c}\text { La eficacia de las tareas cotidianas o habituales de } \\
\text { la empresa }\end{array}$ \\
$\square$ Ha empeorado \\
$\square$ Indiferente \\
$\square$ Ha mejorado un poco \\
$\square$ Ha mejorado bastante \\
$\square$ Ha mejorado mucho
\end{tabular}

Gráfico 7: La detección de problemas

\begin{tabular}{|l|l|}
\hline La detección de problemas internos y áreas de \\
mejora
\end{tabular}$\quad \begin{aligned} & \square \text { Ha empeorado } \\
& \square \text { Indiferente } \\
& \text { poco mejorado un } \\
& \square \text { Ha mejorado } \\
& \text { bastante } \\
& \square \text { Ha mejorado mucho }\end{aligned}$

\subsection{Cuarto bloque: motivos económicos; cuota de mercado y beneficios empresariales}

En este punto se abordan las mejoras económicas y se analiza por un lado la cuota de mercado de la empresa y la posible repercusión de la implantación de la Marca, así como la variación de sus beneficios empresariales.

En cuanto a la cuota de mercado, las empresas encuestadas reflejan diversas opiniones, es una variable muy heterogénea. Hay empresas que han empeorado su cuota de mercado, un $9 \%$, y otras que han mejorado mucho su cuota de mercado, concretamente un 4\%. En el porcentaje más alto, un 39\%, las empresas opinan que la implantación de esta Marca ha sido indiferente en este punto.

En cuanto a los beneficios empresariales, se concluye que el $4 \%$ de las empresas que han empeorado sus beneficios empresariales, el 13\% de las empresas opinan que ha sido indiferente, en el otro extremo se encuentra el $30 \%$ ha mejorado bastante y el 13\% que ha mejorado mucho, pero el mayor porcentaje de opinión, del $40 \%$, es para aquellas que afirman que han mejorado un poco.

Tabla 9: Motivos económicos: cuota de mercado y beneficios empresariales

\begin{tabular}{|c|c|c|c|c|c|}
\hline Valoraciones de la empresa & $\begin{array}{c}\text { Ha } \\
\text { empeorado }\end{array}$ & Indiferente & $\begin{array}{c}\text { Ha } \\
\text { mejorado } \\
\text { un poco }\end{array}$ & $\begin{array}{c}\text { Ha } \\
\text { mejorado } \\
\text { bastante }\end{array}$ & $\begin{array}{c}\text { Ha } \\
\text { mejorado } \\
\text { mucho }\end{array}$ \\
\hline P7. La cuota de mercado & $9 \%$ & $39 \%$ & $26 \%$ & $22 \%$ & $4 \%$ \\
\hline P8. Los beneficios empresariales & $4 \%$ & $13 \%$ & $40 \%$ & $30 \%$ & $13 \%$ \\
\hline
\end{tabular}




\section{Gráfico 8: La cuota de mercado}

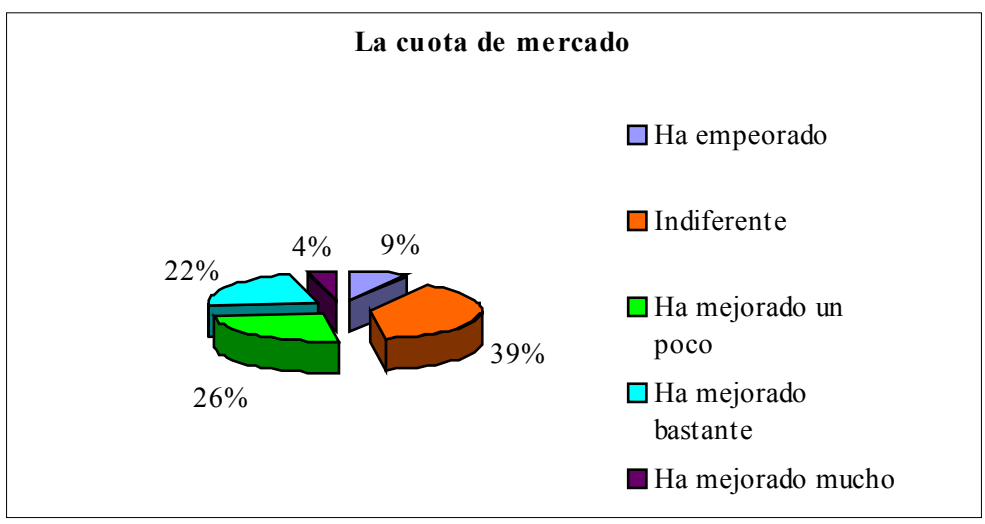

Gráfico 9: Los beneficios empresariales

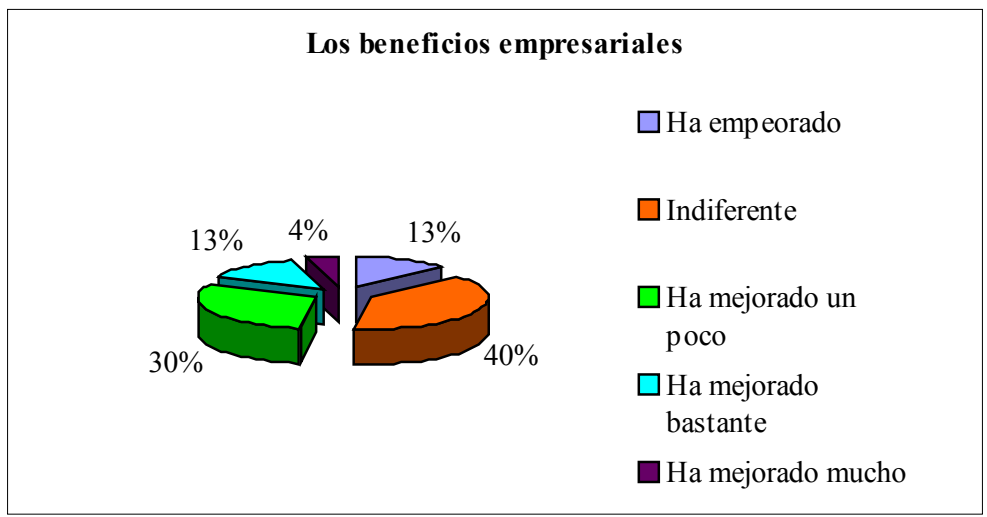

\section{Conclusiones y futuras líneas de investigación}

Visitas a fábricas, industrias, museos industriales, catas, maridajes, visitas a las explotaciones agropecuarias y otras actividades calificadas como Turismo Industrial se están consolidando como un factor multiplicador de los ingresos, bien como canal de venta y promoción complementario al convencional o como alternativa independiente y poderosa para la diversificación del negocio, fuente directa de ingresos y fortalecimiento de marca.

Resulta fundamental ser consciente y consecuente sobre que los efectos e impresiones que la experiencia turística causa en el visitante, repercuten directa y potentemente en la imagen que éste se formará acerca del producto elaborado, influyendo positiva o negativamente en su predisposición a consumir y recomendar el producto y la marca.

Como conclusiones, podemos observar que las preguntas correspondientes al primer bloque, relativo a los motivos basados en la imagen de la empresa y su diferenciación, han sido bien valoradas por las empresas encuestadas. Por ello, podemos afirmar que la marca Q de Turismo Industrial ha significado una mejora en su reconocimiento y prestigio en el mercado.

Las preguntas correspondientes al segundo bloque, son las mejor valoradas, ya que, el 50\% de empresas declara que han mejorado bastante. Este bloque de preguntas se centran en la prestación del servicio y atención al cliente, respectivamente. Con lo que podemos concluir que las empresas encuestadas han mejorado sus procedimientos relativos a la prestación del servicio, así como la atención al cliente. 
En cuanto al tercer bloque de preguntas, referentes a cuestiones de mejora en la gestión en las que se basan las preguntas P5 y P6, no existe una conclusión con alto respaldo en cuanto a la mejora en las gestiones cotidianas de la empresa, ya que el $30 \%$ de las empresas ha empeorado o le resulta indiferente y un $60 \%$ ha notado mejoría en alguna medida (poco, bastante o mucho).

Sobre el cuarto bloque, que estudia los beneficios económicos y la cuota de mercado, podemos decir que no se ha llegado a una respuesta clara o unánime, las valoraciones ofrecidas por las empresas aportan un abanico de opiniones y no podemos llegar a una conclusión. La implantación Marca Q haya supuesto una mejora en la cuota de mercado del $52 \%$ y/o en los beneficios empresariales para el $83 \%$ de las empresas.

A continuación se presenta el porcentaje de mejora obtenido por las empresas en cada pregunta planteada.

\section{Tabla 10: Beneficios y mejora obtenida}

\begin{tabular}{|c|c|}
\hline Pregunta planteada & $\begin{array}{c}\text { Ha mejorado poco, } \\
\text { bastante, mucho }\end{array}$ \\
\hline P1. La imagen de la empresa & $74 \%$ \\
\hline P2. La diferenciación de nuestra empresa respecto a competidores & $74 \%$ \\
\hline P3. La prestación o realización del servicio & $96 \%$ \\
\hline P4. El servicio de atención al cliente & $91 \%$ \\
\hline P5. La eficacia de las tareas cotidianas o habituales & $69 \%$ \\
\hline P6. La detección de problemas internos y áreas de mejora & $87 \%$ \\
\hline P7. La cuota de mercado & $52 \%$ \\
\hline P8. Los beneficios empresariales & $83 \%$ \\
\hline
\end{tabular}

Así, podemos concluir que las preguntas menos valoradas son la P5, que trata sobre la eficacia de las tareas cotidianas, y la P7, que versa sobre la cuota de mercado, al ser valoradas con un $69 \%$ y un $52 \%$, respectivamente. No obstante estos siguen siendo valores que reflejan una mejora en más del $50 \%$ de las empresas encuestadas.

Tabla 11: Beneficios más/menos valorados

\begin{tabular}{|c|c|}
\hline Beneficios más valorados & Beneficios menos valorados \\
\hline $\begin{array}{c}\text { P1. La imagen de la empresa } \\
\text { P2. La diferenciación de nuestra empresa respecto a los } \\
\text { demás competidores } \\
\text { P3. La prestación o realización del servicio } \\
\text { P4. El servicio de atención al cliente } \\
\text { P6. La detección de problemas internos y áreas de } \\
\text { mejora } \\
\text { P8. Los beneficios empresariales }\end{array}$ & $\begin{array}{c}\text { P5. La eficacia de las tareas cotidianas o } \\
\text { habituales de la empresa } \\
\text { P7. La cuota de mercado }\end{array}$ \\
\hline
\end{tabular}

Todos estos resultados comentados con anterioridad se pueden visualizar con mayor detalle en el gráfico que se presentan en este trabajo donde se recoge la distribución de las respuestas de cada uno de los ítems analizados en esta investigación.

Los estudios sobre el turismo industrial son escasos e indispensables para conocer las verdaderas posibilidades de diversificación de la oferta turística de un territorio. Por ello, es conveniente realizar investigaciones con lo que ocurre en el resto de los subsectores turísticos. Como futuras líneas de investigación sería interesante conocer si estas empresas desean mantener la certificación de Turismo Industrial y también si se plantean la implantación/certificación de otros distintitos externos de calidad. También resultaría de interés conocer la evolución de los resultados de este estudio a lo largo del tiempo. 


\section{Gráfico 10: Beneficios de implantación de la Marca $Q$ de Turismo Industrial}

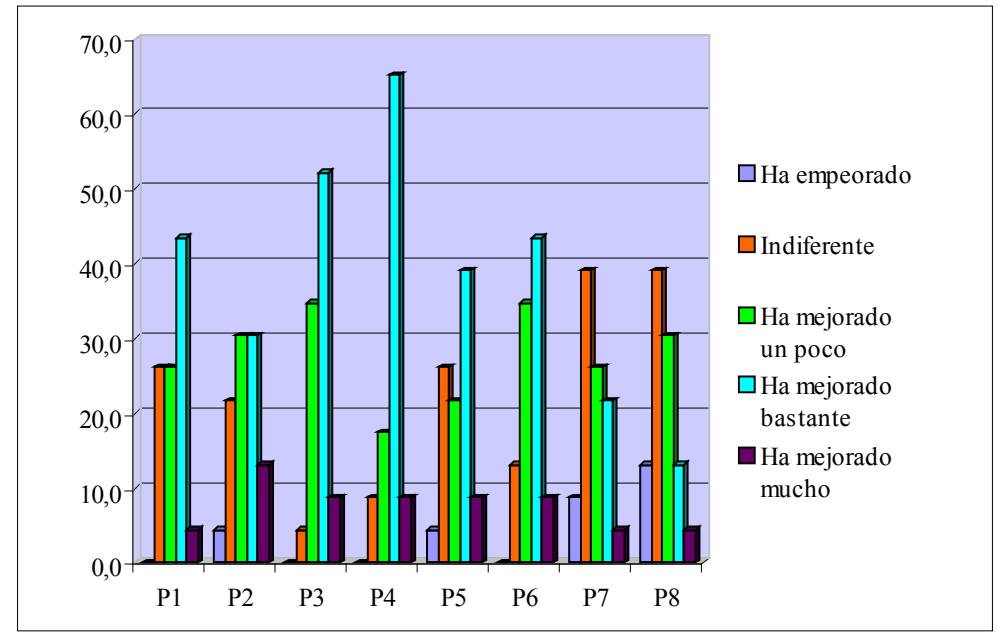

\section{Bibliografía}

\section{Aenor}

2012. “Calidad de Turismo Industrial: UNE 302001:2012”,. Ed. Aenor, Madrid.

Carrasco Fernández, S.

2009. "Procesos de Gestión y Calidad en Hostelería y Turismo", Ed. Vértice, Málaga.

European Foundation For Quality Management

1996. "Autoevaluación directrices para empresas", Ed. E.F.Q.M., Bruselas.

Foley, E.C.

1994. "Winning European Quality”, Ed. E.F.Q.M., Bruselas.

Foronda Robles, C., García López A.M.

2009. "Cuadernos de Turismo", Ed. Universidad de Murcia, Murcia.

Harrington, HJM

1992. "Mejoramiento de los procesos de la empresa", Ed. Díaz de Santos, Madrid.

Horovitz, J.

1987. ¡La calidad del servicio", Ed. Mc. Graw-Hill, Madrid.

Membrado Martínez, J.

2002. "Innovación y mejora continua según el modelo EFQM de excelencia", Ed. Díaz de Santos, Madrid.

Rodríguez, Rodríguez, G.

2009. "Nuevos retos para el turismo", Ed. Netbiblo, A Coruña.

Yepes Piqueras, V.

2009. "Incorporación de la gestión de calidad en las empresas y destinos turísticos", Ed. Visión Libros, Madrid.

\section{Páginas Web consultadas}

www.aenor.es

www.icte.es

www.turismoindustrial.org

www.turgalicia.es

www.doriasbaixas.com

www.hispanobodegas.com 